\title{
Aplicación de la Bioquímica y Biología Molecular en el Grado de Enfermería a través del aprendizaje basado en problemas
}

CRISTINA TOUS RIVERA

Centro Universitario de Enfermería Cruz

Roja, adscrito a la Universidad de Sevilla

ctous@us.es

ORCID: https://orcid.org/0000-0003-4007-2001

D.O.I.: http://dx.doi.org/10.12795/JDU.2018.i01.17

Pp.: $307-320$

\section{Resumen}

El CMD docente (CMD) se ha realizado en la asignatura de 1er curso "Bioquímica y Biología Molecular". Se ha desarrollado incluyendo el temario correspondiente al bloque de Proteínas. Han participado 56 estudiantes. El trabajo se ha realizado, inicialmente individual y fuera del aula, aunque algunos optaron por hacerlo conjunto, en subgrupos y con un aprendizaje colaborativo. Se han empleado los mapas de contenidos y se ha evaluado el CMD mediante el uso de escaleras de aprendizaje. Los resultados muestran, en general, una adquisición adecuada de los conocimientos trabajados y han sido bastante satisfactorios para el alumnado. 
Palabras claves: Bioquímica y Biología Molecular, Grado de Enfermería, Docencia universitaria, Experimentación docente universitaria, Aprendizaje Basado en Problemas

\section{Breve descripción del contexto}

La asignatura donde se ha realizado el CMD es "Bioquímica y Biología Molecular" del primer curso del Grado de Enfermería, en el Centro Universitario de Enfermería Cruz Roja, adscrito a la Universidad de Sevilla. Es una asignatura cuatrimestral de 6 créditos ECTS, que se imparte en el primer cuatrimestre del curso y es de formación básica. La docencia presencial se distribuye en 50 horas de clases teóricas y 10 horas de clases prácticas. Los estudiantes son en su mayoría de bachillerato de ciencia aunque hay una proporción alta que procede de ciclos formativos teniendo un nivel inferior en conocimientos de biología. El grupo está formado por aproximadamente 75 estudiantes, de los cuales suelen asistir a clase teóricas 65 de ellos. Las clases prácticas se dividen en 5 grupos de unos 15 estudiantes cada uno. El aula donde se desarrollan las clases es grande y espaciosa pero no dispone de un mobiliario adecuado para la movilidad del alumnado. El CMD ha tenido una duración de 14 horas y se ha desarrollado en 7 sesiones teóricas de grupo completo entre las fechas 18 de octubre y 13 de noviembre.

Jornadas de Formación e Innovación Docente del Profesorado | № 1 (2018) Esta obra se distribuye con la licencia Creative Commons 


\section{Diseño previo del CMD}

\section{Modelo metodológico consolidado}

Hasta ahora, estos temas se abordaban desde seminarios que al azar preparaban algunos estudiantes. Ellos tenían unos 20 minutos para explicar estos temas a los demás compañeros. Tras su exposición, había una tanda de preguntas para resolver las dudas que se planteaban.

Este año el tema de las proteínas, su función y estructura se ha desarrollado con más profundidad, ya que con el método de seminarios los conceptos se quedaban muy superficiales e incluso algunos eran escasos.

\section{Modelo metodológico ideal}

El modelo metodológico que se ha seguido en el CMD consiste en la resolución en clase de unas preguntas ( $P$ ) dadas con anterioridad a los estudiantes y que ellos han tenido que trabajar fuera del aula. Al menos con dos días de antelación a la clase, enviando sus respuestas al correo de la profesora. Las respuestas a dichas preguntas englobaban la mayor parte de los conceptos que se querían transmitir.

Al iniciar la clase, se dejó unos 5 minutos para aclarar dudas de la clase anterior o problema general (D). En este CMD, se incluye el trabajo en clase de cada respuesta en grupos de $4(\mathrm{G})$. Durante los 30 minutos ellos leyeron sus respuestas y llegaron a un consenso en la que incluían toda la información que consideran que diera respuesta a la pregunta formulada. Entre los 4 elegían a un secretario que anotaba y después exponía. Se les propuso la participación voluntaria para leer sus respuestas y comparar 
con los demás la información obtenida (R). Tras sus aportaciones y finalizado el tiempo permitido (30 min) para dar respuesta a la primera pregunta, se les hizo un breve resumen de lo que habían expuesto y se les amplió la información que no se hubiera mencionado o faltase en dicha respuesta. Al igual, se aclararon conceptos que hubieran aparecidos durante el debate y que ellos estuvieran confusos o incluso erróneos (E). Seguidamente, se pasaba a resolver la siguiente pregunta con la misma metodología de trabajo. Para finalizar la clase se recordó a los estudiantes (d) cuál sería la próxima tarea a realizar.

En las cuatro siguientes sesiones la metodología que se aplicó fue similar, cambiando las preguntas que se resolvían cada día. Para la última sesión se realizó un resumen general de todos los conceptos adquiridos y para ello realicé el juego del PASAPALABRAS. Elaboré un rosco de preguntas donde se incluían todo lo visto en las sesiones anteriores. Un estudiante elegido al azar de cada grupo representó al equipo y fue el que respondió a las preguntas. Se realizó una a cada equipo y el que no la supiera pasaba la palabra al siguiente equipo. Aquél equipo que respondió a más preguntas fue el ganador. De esta forma el repaso general fue más dinámico.

\section{Mapa de contenidos.}

En el presente CDM se presentan los contenidos que quería enseñar a los estudiantes (García, Porlán y Navarro, 2017), mediante el desarrollo de las diferentes cuestiones relacionadas con las proteínas. Desde su síntesis, organización, estructura y función. Los contenidos conceptuales están resaltados en rojo, los procedimentales en Amarillo y los valores en azul. La figura 1 reúne los contenidos de aprendizaje: 


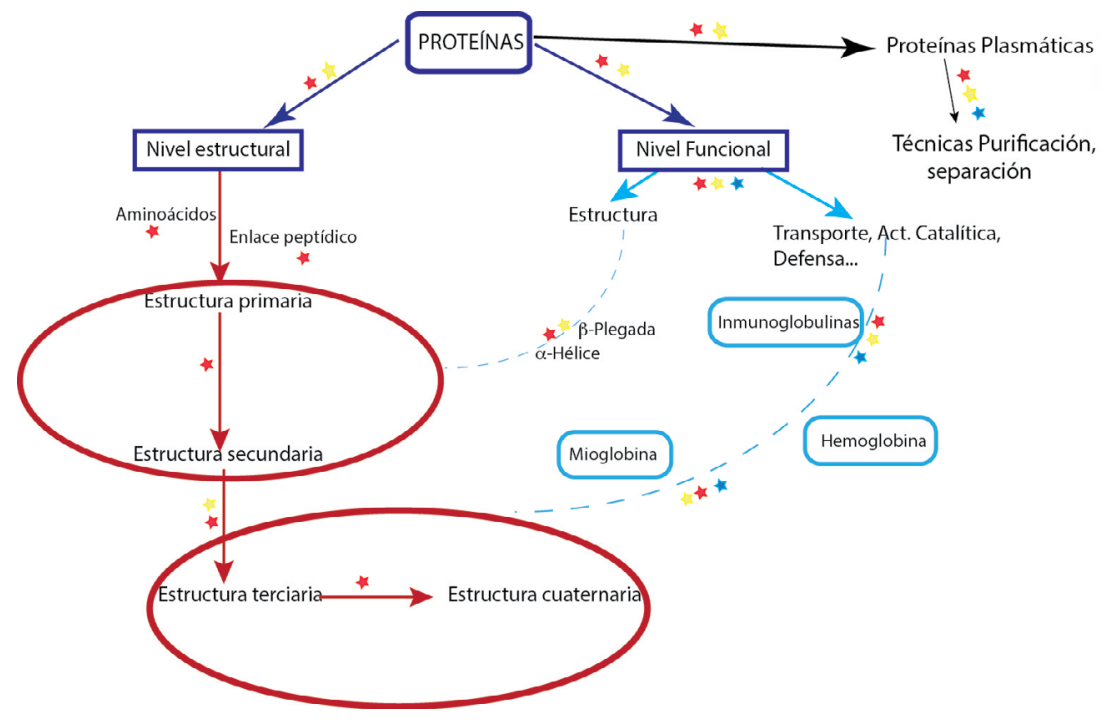

Figura 1. Mapa de contenidos.

Los conceptos fundamentales los marca el color de cada flecha. La Estrella roja determina los conceptos a adquirir (Estructura de las proteínas, aminoácidos y enlace peptídico, función de las proteínas). La Estrella amarilla indica conceptos procedimentales (relacionar la función de una proteína con su estructura). La Estrella azul indica valores (ser consciente de la importancia de la proteínas en el ser vivo).

\section{Secuencia de actividades antes de aplicar el CMD.}

A continuación se expone la secuencia de actividades (tabla 1).

Jornadas de Formación e Innovación Docente del Profesorado | № 1 (2018)

(C) Esta obra se distribuye con la licencia Creative Commons Reconocimiento-NoComercial-SinObraDerivada $\quad 4.0$ 
Tabla 1.

Secuencia de actividades antes de aplicar el CMD.

\begin{tabular}{|c|c|c|}
\hline & 7 sesiones del CMD (inicia el 18/10/2018-13/Nov/2018) & \\
\hline$P$ & $\begin{array}{l}\text { - En la clase anterior al inicio de la clase de mejora se le } \\
\text { pasará un cuestionario con las preguntas que más tarde } \\
\text { trabajarán fuera del aula. Se les informa que es indivi- } \\
\text { dual y que no tendrá ningún carácter evaluador. } \\
\text { - } \text { Las preguntas se les enviará por correo a todos. Se les } \\
\text { comenta que deben profundizar en sus respuestas, que } \\
\text { una respuesta de tan solo } 3 \text { líneas no se considera su- } \\
\text { ficiente. Enviar las respuestas dos días antes de la si- } \\
\text { guiente clase. }\end{array}$ & $20^{-}$ \\
\hline D & $\begin{array}{l}\text { - Se comienza la clase dejando unos minutos para resolver } \\
\text { dudas de la clase anterior. Utilizo las imágenes y power- } \\
\text { point para aclarar las dudas. Si hay pocas preguntas, in- } \\
\text { sistir en que miren lo dado el día o semana anterior. }\end{array}$ & 5 \\
\hline G & $\begin{array}{l}\text { - Se reunirán en grupos de } 4 \text { para responder a la primera } \\
\text { pregunta. Deberán cada uno aportar la información que } \\
\text { tengan para llegar a una común. Elegirán un secretario } \\
\text { que recoja la información. El profesor estará entre los } \\
\text { grupos para resolver dudas y ver como interaccionan en- } \\
\text { tre ellos. }\end{array}$ & $20^{-}$ \\
\hline $\mathrm{R}$ & $\begin{array}{l}\text { - Se piden voluntarios para que lean la respuesta a la } \\
\text { primera pregunta Tras la participación de } 5 \text { o } 6 \text { grupos } \\
\text { podrán ir aportando ideas o conceptos que crean que } \\
\text { deban cambiar o ampliar. La participación del profesor } \\
\text { consistirá en preguntar que añadirían a las respuestas } \\
\text { dadas para intentar generar un diálogo y que lleguen a } \\
\text { una respuesta común y consensuada. }\end{array}$ & $30^{-}$ \\
\hline E & $\begin{array}{l}\text { - Tras los } 30 \text { minutos, les hago un breve resumen de la res- } \\
\text { puesta que han ido construyendo, para afianzar los co- } \\
\text { nocimientos adquiridos y se ampliará la información si } \\
\text { es necesario. Para ello se empleará como soporte diapo- } \\
\text { sitivas de powerpoint. En las explicaciones se profundi- } \\
\text { zará en los contenidos teóricos que dan respuesta a las } \\
\text { preguntas planteadas. }\end{array}$ & $10^{-}$ \\
\hline$G_{n}$ & $\begin{array}{l}\text { - Se volverá a reunir en grupo y se pasará a responder la } \\
\text { segunda pregunta }\end{array}$ & $20^{-}$ \\
\hline
\end{tabular}

Jornadas de Formación e Innovación Docente del Profesorado I № 1 (2018) Esta obra se distribuye con la licencia Creative Commons Reconocimiento-NoComercial-SinObraDerivada Internacional (CC BY-NC-ND 4.0.) 


\begin{tabular}{|c|c|c|c|}
\hline$R_{n}$ & $\begin{array}{l}- \\
- \\
-\end{array}$ & $\begin{array}{l}\text { Se pasará a responder la segunda pregunta planteada. } \\
\text { Se pedirán voluntarios para que lean sus respuestas y se } \\
\text { animará a los mas callados a que den su opinión. } \\
\text { El profesor, tras } 3 \text { o } 4 \text { intervenciones, podrá ir esquema- } \\
\text { tizando en la pizarra las ideas que van dando, para que } \\
\text { puedan ir siguiendo su propio hilo conductor. }\end{array}$ & $30^{-}$ \\
\hline$E_{n}$ & & $\begin{array}{l}\text { Una vez hayan aclarada la respuesta, el profesor volverá } \\
\text { a hacer un resumen de las conclusiones y se ampliará la } \\
\text { información mas relevante. Para ello se utilizarán diapo- } \\
\text { sitivas en powerpoint. }\end{array}$ & $10^{-}$ \\
\hline \multirow[t]{2}{*}{$d$} & - & $\begin{array}{l}\text { Los últimos } 2 \text { min antes de finalizar, se recuerda que en } \\
\text { la siguiente sesión se van a responder las siguientes pre- } \\
\text { guntas. Se les anima a revisar lo que han respondido por } \\
\text { si quieren incluir nueva información o modificar algo. Tas } \\
\text { la segunda sesión les recuerdo que tendrán que enviar } \\
\text { por correo las respuestas a las preguntas que se trabaja- } \\
\text { rán en la semana siguiente. }\end{array}$ & $2^{-}$ \\
\hline & - & Última sesión del CMD & \\
\hline \multirow[t]{2}{*}{ RG } & - & $\begin{array}{l}\text { Tras finalizar las cuestiones se hará un resumen gene- } \\
\text { ral de todas ellas. Se hará conjunto entre los estudian- } \\
\text { tes y el profesor. } \\
\text { Al terminar se les Planteará hacer el juego de } \\
\text { pasapalabras. }\end{array}$ & $60^{-}$ \\
\hline & & $\begin{array}{l}\text { Tras termina el juego se les volverá a pasar el cuestiona- } \\
\text { rio inicial para que vuelvan a rellenarlo con los conoci- } \\
\text { mientos adquiridos en estas } 5 \text { sesiones. }\end{array}$ & 45 \\
\hline
\end{tabular}

\section{Cuestionario inicial y final.}

Las preguntas claves representan a los conceptos fundamentales que se quieren transmitir, el color indica a que concepto fundamental hace hincapié:

1. ¿Qué es una proteína?, ¿cómo las clasificarías, por su estructura, por su función, por ambas?, Razónalo.

2. Que entiendes por niveles estructurales de las proteínas. Descríbelos.

3. ¿Cuál es la unidad mas pequeña que forman a las proteínas? 
3.1. Esta unidad tiene una importante función biológica, ¿sabrías decir por qué?

4. La disposición tridimensional de una proteína, ¿a que se corresponde?

¿Cómo adquiere dicha estructura?

5. ¿La Mioglobina y la Hemoglobina tienen igual función?, ¿por qué?. Enumera las similitudes y diferencias.

6. Di alguna proteína muy abundante que solo tenga función estructural. ¿Y otra que sea de defensa?. Di como son las estructuras de ambas.

7. El plasma sanguíneo está formado por muchas proteínas con diversas funciones. ¿Sabrías explicar las mas abundantes e importantes?

8. El estudio de las proteínas nos ayudan a identificar enfermedades o patologías desconocidas e incluso seguir enfermedades ya conocidas. ¿Existen técnicas de laboratorio que nos permiten obtener dichas proteínas?. ¿Cuáles?.

\section{Aplicación del CMD.}

\section{Relato resumido de las sesiones}

Las diferentes sesiones, mas o menos fueron desarrollándose de forma similar. Las sesiones comenzaban dando 5 minutos para que preguntasen dudas surgidas en el temario dado en la clase anterior. Este año, he notado que es una clase que no aprovecha ese tiempo.

Como en la última clase que tuve con ellos ya les había explicado la metodología a seguir, tan solo tuve que hacer un breve recordatorio. Les agradeci el trabajo que habian realizado fuera del aula, y les pasé el cuestionario inicial que realizaron en 8 minutos. Inmediatamente comenzaron poniéndose en grupo para trabajar la primera pregunta. 
Me sorprendió lo rápido que se unieron en grupos de 4 o 6 de forma excepcional, y lo bien que trabajaron. Pasado el tiempo de puesta en común, comenzaron a dar sus respuestas mientras yo esquematizaba en la pizarra. Fueron bastante ágiles, seguros de sus respuestas y entre ellos se aclaraban las dudas. Cuando terminaron, les hice un resumen de lo que ellos habían expuesto, sin tener que añadir nada.

Inmediatamente pasaron a poner en común la segunda pregunta. Como era bastante sencilla, se realizó en menor tiempo y se contestó por otros grupos que no habían participado anteriormente.

En esta pegunta si había pequeñas dudas que tuve que resolver al final.

Cuando terminaron hice un breve resumen de todo lo visto en el día. Sus caras eran de cansados/saturados.

Les recordé el trabajo a realizar para la siguiente clase y me despedí deseándoles una buena tarde.

La siguiente sesión de CMD comenzó igual, esta vez si tuvieron dudas relacionadas en cómo ampliar la información que respondía a cada pregunta. Les hice ver que no hay límites, que el saber no ocupa lugar y que debían de responder de forma extensa pero sabiendo que responden. Quise introducir un cambio en la metodología y en vez de ser yo la que escribía el resumen/esquema en la pizarra, sería uno del grupo que daba la respuesta.

Eso les gustó y empezaron a solicitar ser los primeros en dar las respuesta. Las 2 preguntas que tocaban responder eran mas largas así que necesitaron mas tiempo para contestarla. Mi sorpresa fue, que no saben hacer esquemas mientras escuchan, el que escribía en la pizarra ya tenia su esquema hecho y lo copiaba en la pizarra, mientras que el que leía iba a otro ritmo. Así que mi idea de intentar agilizar el momento esquema fue peor para mi, pero ellos estaban encantaos.

Respondieron bastante bien a las preguntas planteadas y al final de la clase utilicé mi powerpoint para resumirles todo lo visto hasta entonces. 
Al terminar la clase, tras recordar de nuevo la tarea a realizar fuera del aula, varias alumnas me comentaron que aunque la clase era intensa y estaban en continua atención, la mayoría estaban encantados con las 2 clases que habíamos dado.

Las demás sesiones fueron similares, lo único que me suponía cambios era la temporización, ya que al ver a la mayoría de los grupos entregados en llegar a una respuesta perfecta, les daba mas tiempo de lo estipulado, con el consiguiente retraso para las demás sesiones.

La última sesión, que originalmente era para realizar un resumen general y realizar un juego, comenzó terminando de contestar la última cuestión.

Tras finalizar, hice un resumen de todo lo dado y les explique el juego de PASAPALABRAS. Un representante de cada 2 grupos mas o menos era el portavoz ( eran 8 participantes) y por tanto el que daba la respuesta, los demás compañeros le podían ayudar.

Al principio, algunos grupos no querían participar pero al decir que el premio eran bombones, se animaron.

Resultó divertido e incluso competitivo, pero me dejó poco tiempo para volver a pasar el cuestionario. Aún así, ellos se sentaron rápidamente y realizaron el cuestionario perfectamente (Finkel, 2008).

\section{Evaluación del aprendizaje de los estudiantes.}

\section{Escaleras de aprendizaje.}

Tras la realización del CMD se analizaron los resultados de aprendizaje de los estudiantes. Para ello se compararon los resultados de los dos cuestionarios, inicial y final, que contenían las preguntas que posteriormente se habían trabajado durante el ciclo. Se ha analizado una muestra de 43 cuestionarios individuales, de los 65 que se recogieron. A continuación se muestran las escaleras de 
conocimiento y aprendizaje correspondientes a las cuestiones mas representativas.

Pregunta 1. ¿Qué es una proteína?, ¿cómo las clasificarías, por su estructura, por su función, por ambas?, Razónalo (figuras 2 y 3 ).

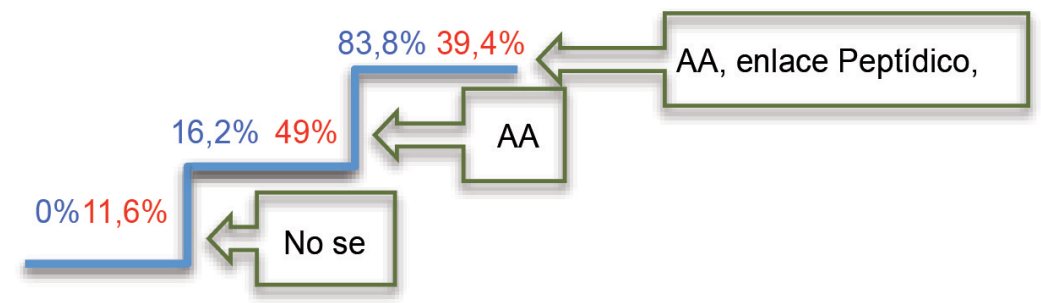

Figura 2. Escalera de aprendizaje de las preguntas sobre las proteínas.

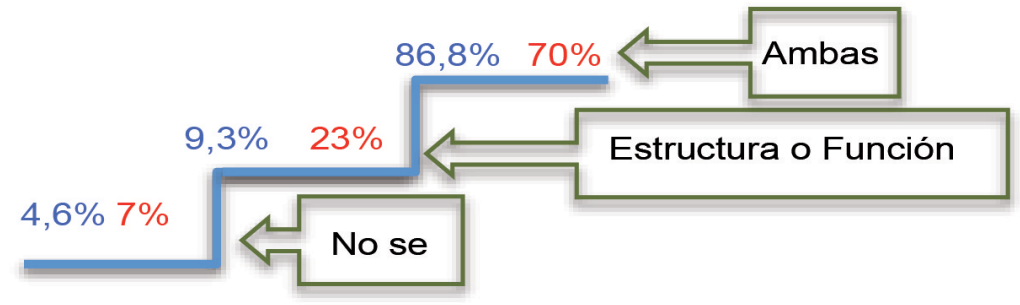

Figura 3. Escalera de aprendizaje de las preguntas sobre la estructura de las proteínas.

El resultado inicial indica que el $49 \%$ de los estudiantes saben que las proteínas están, formadas por aminoácidos. Sin embargo hay un casi $12 \%$ de ellos no responden. Tras el CMD, el $84 \%$ de los estudiantes tienen claro las proteínas están formadas por aminoácidos unidos por enlace peptídico. En la segunda cuestión, partíamos de un 70\% de estudiantes que saben que las proteínas se clasifican tanto por su estructura y su función. Tras el CDM este conocimiento aumentó al 87\% del alumnado.

Pregunta 5. ¿La Mioglobina y la Hemoglobina tienen igual función?, ¿por qué?. Enumera las similitudes y diferencias (figura 4).

Jornadas de Formación e Innovación Docente del Profesorado I № 1 (2018) Esta obra se distribuye con la licencia Creative Commons 


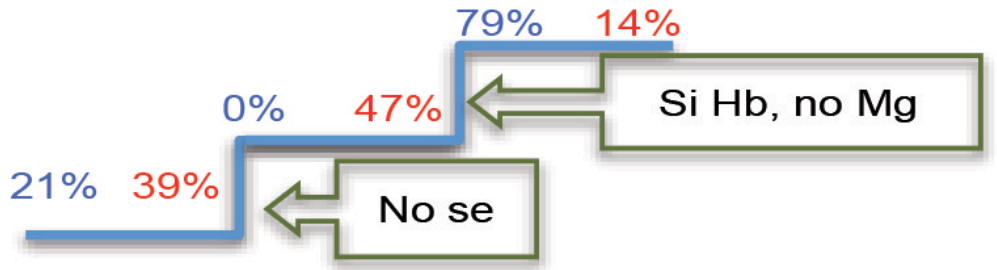

Figura 4. Escalera de aprendizaje de las preguntas sobre la Mioglobina y la Hemoglobina.

Antes de comenzar el CMD, el $47 \%$ de los estudiantes sabían cuál era la función de la Hemoglobina pero no de la mioglobina. Tras el ciclo el 79\% tiene claro las diferencias y similitudes de ambas proteínas.

Pregunta 6. Di alguna proteína muy abundante que solo tenga función estructural. ¿Y otra que sea de defensa?. Di como son las estructuras de ambas (figura 5).

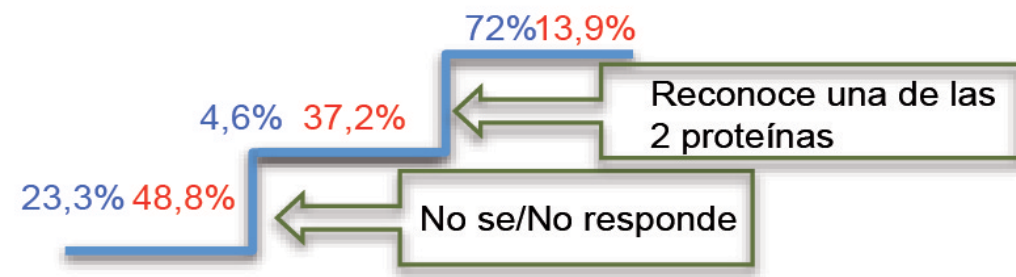

Figura 5. Escalera de aprendizaje de las preguntas sobre las estructuras de las proteínas.

En esta cuestión, hay un porcentaje elevado de estudiantes que no saben contestar. Tras el CMD, el $72 \%$ del alumnado sabe nombrar e identificar proteínas estructurales como el colágeno y proteínas de defensa, como las Inmunoglobulinas.

Pregunta 8. El estudio de las proteínas nos ayudan a identificar enfermedades o patologías desconocidas e incluso seguir enfermedades ya conocidas. ¿Existen técnicas de laboratorio que nos permiten obtener dichas proteínas?. ¿Cuáles? (figura 6). 


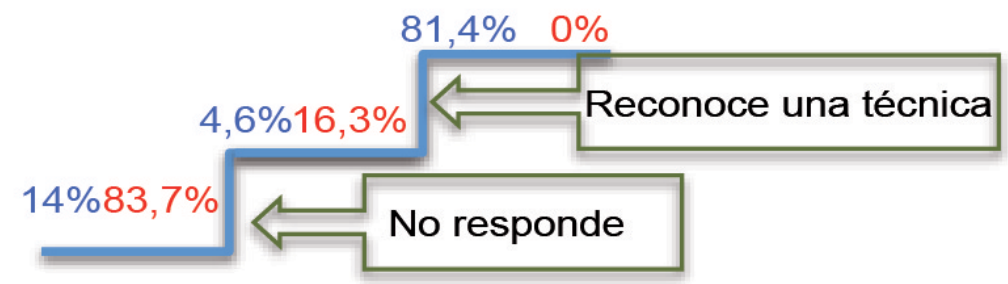

Figura 6. Escalera de aprendizaje de las preguntas sobre las técnicas de laboratorio y proteínas.

\section{Evaluación del CMD}

Para la evaluación del CMD se ha empleado la observación directa de los estudiantes y el cuaderno de campo. En este se ha anotado como se han desarrollando cada una de las actividades que se han realizado, al igual que las impresiones percibidas. Además, al finalizar la última sesión se le distribuyó un cuestionario de satisfacción.

Este año me he encontrado con el problema que al ser las sesiones mas largas de lo esperado, el cuestionario de satisfacción no pude entregarlo para que me lo rellenasen, así que se lo subí a la plataforma virtual para que ellos lo rellenasen y lo entregasen anónimamente.

El problema ha sido que lo han entregado muy pocos estudiantes, por lo que la evaluación del profesor no he podido realizarla, aunque si aplicar los comentarios y sugerencias que me han hecho los pocos estudiantes que lo han rellenado.

\section{Cuestiones a mantener y cambios a introducir}

Me quedo con la gran participación de lo estudiantes y el interés por resolver los problemas planteados. La participación no sólo se ha observado en el trabajo fuera del aula, sino también, en sus intervenciones en clase y las discusiones generadas entre ellos. El detalle de no coger apuntes mientras hago el resumen, me ha demostrado 
que la asimilación de los conceptos ha sido amplia y que han sabido conectar la pregunta con la respuesta.

Quiero seguir incluyendo la dinámica del trabajo fuera del aula y después en clase aunque intentaré variar las actividades para evitar entrar en monotonía. He visto que ser ellos parte activa les motiva y esto hace que todo sea dinámico y sencillo (Bain, 2007). Estoy muy contenta con el resultado.

\section{Bibliografía}

Bain, K. (2007). Lo que hacen los mejores profesores de Universidad. Valencia: Publicaciones de la Universitat de Valéncia.

Finkel, D. (2008). Dar clase con la boca cerrada. Valencia: Publicaciones de la Universitat de Valéncia.

García, E., Porlán, R. y Navarro, E. (2017). Los fines y los contenidos. En R. Porlán (Coord.), Enseñanza universitaria. Cómo mejorarla (55-72). Madrid: Ediciones Morata.

Jornadas de Formación e Innovación Docente del Profesorado I № 1 (2018) Esta obra se distribuye con la licencia Creative Commons 